\title{
Risk Factors for Recurrent L4-5 Disc Herniation After Percutaneous Endoscopic Transforaminal Discectomy: A Retrospective Analysis of 654 Cases
}

This article was published in the following Dove Press journal: Risk Management and Healthcare Policy

\author{
Meng Kong $\mathbb{D}^{1,2, *}$ \\ Derong $\mathrm{Xu}^{\mathrm{l}}$ ** \\ Changtong $\mathrm{Gao}^{3}$ \\ Kai Zhu' \\ Shuo Han ${ }^{1,2}$ \\ Hao Zhang (D) ${ }^{1,2}$ \\ Chuanli Zhou' \\ Xuexiao $\mathrm{Ma}^{\prime}$
}

'Department of Spinal Surgery, The Affiliated Hospital of Qingdao University, Qing'dao, Shandong Province 266000,

People's Republic of China; ${ }^{2}$ Department of Medicine, Qingdao University,

Qing'dao, Shandong Province 266000, People's Republic of China; ${ }^{3}$ Minimally Invasive Interventional Therapy Center, Qingdao Municipal Hospital, Qing'dao, Shandong Province 266000, People's Republic of China

*These authors contributed equally to this work
Background: Percutaneous endoscopic lumbar discectomy (PELD) is an increasingly applied minimally invasive procedure that has several advantages in the treatment of lumbar disc herniation (LDH). However, recurrent LDH (rLDH) has become a concerning postoperative complication. It remains difficult to establish a consensus and draw reliable conclusions regarding the risk factors for $\mathrm{rLDH}$.

Purpose: This retrospective study aimed to investigate the risk factors associated with rLDH at the L4-5 level after percutaneous endoscopic transforaminal discectomy (PETD).

Methods: A total of 654 patients who underwent the PETD procedure at the L4-5 level from October 2013 to January 2020 were divided into a recurrence (R) group $(n=46)$ and a nonrecurrence $(N)$ group $(n=608)$. Demographic and clinical data and imaging parameters were collected and analyzed using univariate and multiple regression analyses.

Results: The current study found a 7\% rate of rLDH at the L4/5 level after successful PETD. Univariate analysis showed that older age, high BMI, diabetes mellitus history, smoking, large physical load intensity, moderate disc degeneration, small muscle-disc ratio (M/D), more fat infiltration, large sagittal range of motion (sROM), scoliosis, small disc height index (DHI), small intervertebral space angle (ISA), and small lumbar lordosis (LL) were potential risk factors $(\mathrm{P}<0.10)$ for LDH recurrence after PETD at the L4-5 level. Multivariate analysis suggested that high BMI, large physical load intensity, moderate disc degeneration, small M/D, more fat infiltration, large sROM, small ISA, and small LL were independent significant risk factors for recurrence of LDH after PETD.

Conclusion: Consideration of disc degeneration, $\mathrm{M} / \mathrm{D}$, fat infiltration of the paravertebral muscles, sROM, ISA, LL, BMI, and physical load intensity prior to surgical intervention may contribute to the prevention of rLDH following PETD and lead to a more satisfactory operative outcome and the development of a reasonable rehabilitation program after discharge.

Keywords: percutaneous endoscopic transforaminal discectomy, recurrent LDH, risk factor

\section{Introduction}

Correspondence: Xuexiao Ma

Tel +8618661807895

Email ma_xuexiao@I26.com

Chuanli Zhou

Department of Spinal Surgery, The Affiliated Hospital of Qingdao University,

No. 59, Hai Er Road, Qing'dao, Shandong

Province 266000, People's Republic of

China

Tel +86 I8661809796

Email justin_5257@hotmail.com
Percutaneous endoscopic lumbar discectomy (PELD) is a minimally invasive procedure that is increasingly used by spinal surgeons in the treatment of lumbar disc herniation (LDH). PELD has an effectiveness similar to that of conventional surgery, and it has several advantages over open discectomy, including smaller incisions, less paravertebral muscle injury and bony structure destruction, faster recovery, and fewer postoperative complications. ${ }^{1,2}$ However, with the widespread use of this technique, recurrence of disc herniation has become a concerning postoperative complication. ${ }^{3}$ 
The rate of recurrent LDH (rLDH) after successful PELD ranges from 0 to $12.5 \%{ }^{4,5}$ Recurrent $\mathrm{LDH}$ often requires secondary surgery, which is complicated due to epidural fibrosis and scarring, and it results in the psychological suffering of patients. ${ }^{6}$

Surgeons using PELD must pay more attention to potential risk factors for $\mathrm{rLDH}$, which would provide the necessary basis for the selection of appropriate patients and the identification of patients who warrant further study to delineate an optimal personalized therapy. Some of the most often-mentioned risk factors for recurrence are older age, obesity, degree of disc degeneration, location of herniation, and the learning curve of the surgeon. ${ }^{7,8}$ To the best of our knowledge, very few previous in-depth investigations reported estimated risk factors for $\mathrm{rLDH}$ after PELD. A limited number of studies have described the effects of biomechanical elements and anatomical structures, such as disc height index (DHI), intervertebral space angle (ISA), sagittal range of motion (sROM), paravertebral muscles, and structural modification parameters, on rLDH.

Previous research suggests that the biomechanical exposition of each lumbar disc level differs, ${ }^{9,10}$ and some studies indicated that the L4-5 segment showed a comparatively higher incidence of $\mathrm{rLDH}$ than other levels. ${ }^{11,12}$ The number of patients who underwent percutaneous endoscopic transforaminal discectomy (PETD) at the L4-5 segment in our department was overwhelming, and percutaneous endoscopic interlaminar discectomy (PEID) was routinely applied at the L5-S1 level. L5-S1 has special anatomical associations with the pelvic structures. Therefore, we performed a single-centerbased retrospective study of patients treated with PETD at the L4-5 level to sufficiently identify comprehensive risk factors, especially biomechanical factors, associated with L4-5 rLDH after PETD and provide detailed evidence-based data for use in preoperative evaluations and the selection of appropriate patients.

\section{Patients and Methods}

\section{Patient Selection}

A retrospective study of patients treated with PETD for L4-5 disc herniation in our department was performed. All radiological and relevant clinical data during the perioperative period and postoperative follow-up were collected from the regular registry and used to identify patients with $\mathrm{rLDH}$. The study included the period from October 2013 to January 2020.
The computerized database was searched to identify standard candidates who matched the inclusion criteria: 1) patients who had undergone a surgical procedure using the transforaminal approach at the L4-5 level; 2) patients whose postoperative MRI showed complete removal of the protruded disc; 3) patients who experienced a successful symptom-free interval of at least 2 weeks after PETD; and 4) patients who were followed up for at least 6 months. The following exclusion criteria were used: 1) patients who experienced persistent symptoms and no pain-free interval after surgery were verified to have remnant fragments using MRI, and ultimately required a subsequent procedure in the hospital (these were not defined as recurrence of disc herniation but as operative failure); 2) patients with recurrent disc herniation that was not on the same level as the previous PETD; 3) patients who did not participate in follow-up; 4) patients who suffered from other thoracolumbar spinal disorders, such as infection, tumor, ankylosing spondylitis, or rheumatoid arthritis; and 5) patients with a history of discectomy or vertebral augmentation at the index level or who had received other lumbar surgeries within the three adjacent spinal levels.

The following indications for PETD application were used: 1) disc herniation and dural sac or nerve root compression confirmed using MRI; 2) distribution of radiculopathic lower limb symptoms consistent with nerve root compression on MRI; and 3) no obvious spondylolisthesis or spinal stenosis.

According to the criteria described in a previous study, ${ }^{12}$ new symptomatic relapsed disc herniation was diagnosed as follows: 1) radiculopathic lower limb pain that recurred after a successful symptom-free interval of at least 2 weeks; and 2) follow-up MRI that showed newly developed disc protrusion at the previously operated site, regardless of any ipsilateral or contralateral herniation. The subjects in this study who experienced rLDH all underwent reoperation. All of the postoperative data of the reoperation were excluded from this research.

\section{Study Groups, Clinical and Radiographic Examinations}

Patients who met the criteria were divided into two groups: 1) a recurrence group (R) and 2) a nonrecurrence group $(\mathrm{N})$. Our department routinely recorded information on general factors (eg, age, gender, body mass index (BMI), duration of symptoms, hypertension history, 
diabetes mellitus, smoking, and alcohol use) and performed lumbar imaging examination (X-ray in the anteroposterior and flexion-extension lateral positions, three-dimensional computed tomography (3D-CT), and magnetic resonance imaging (MRI)) before surgery.

\section{Surgical Technique}

The present study used transforaminal endoscopic approaches (outside-in technique) for the surgical management of lumbar disc herniation. The method involved the sequential insertion of the working channel beside the epidural space (out of the disc space) followed by a subsequent approach of the disc space.

The patient was placed in the prone position on a hyperkyphotic frame on top of a radiolucent table. The skin entry point was selected according to the location of the herniation, the foraminal dimension, and the body size of the patient. After the administration of local anesthesia, the following steps were performed: 1) an 18-gauge spinal needle was placed at a $25-35^{\circ}$ angle to the horizontal in the anterior-posterior view, the superior articular process was reached under intermittent fluoroscopic guidance, and additional local anesthesia was injected; 2) a guidewire was inserted into the spinal needle; 3 ) the spinal needle was removed, and a small skin entry incision was made at the previously marked point; 4) a taper-ended tubularaccess cannula was inserted into the skin incision through the guidewire to subsequently expand the soft tissues; 5) the superior articular process was reduced, and the intervertebral foramen was enlarged using a trephine; 6) a beveled work channel was placed along the path of the trephine, and a transforaminal endoscope was sited through the superior articular process and outside the intervertebral disc; 7) the herniated nucleus pulposus was removed, and the tissues were pressed with various grasping forceps or abrasive drilling after identification of the structures; and 8) annulotomy was selectively performed to achieve sufficient removal of the herniated disc and relief of symptoms. A change in the color of the disc from pale to pink and a movement range of the nerve roots of 2-3 $\mathrm{mm}$ were used as criteria for completion of the surgery. An expert surgeon with sufficient experience and competency in PETD performed all endoscopic surgeries.

\section{Postoperative Management}

Patients were encouraged to walk 1 day after the procedure with the protection of waist support. Full activity was allowed 1 month postoperatively. At this time, the strength of the fibrotic scar formed at the disc gap was sufficient. Lumbar MRI and 3D-CT were routinely performed 1 day before discharge and at 3 months in the clinic after PETD. Thereafter, all the patients were contacted by telephone regularly at 6-month intervals after the procedure.

During the follow-up period, all of the subjects completed a self-administered questionnaire that included eight questions based on the Stockholm Public Health Questionnaire (SPHQ) to evaluate the mean physical load intensity after returning to full activity (Table S1). ${ }^{13}$ The duration, frequency, and amplitude of all response scales were measured at an ordinal level of -4 to 5 points. The total physical load intensity score was obtained by summing the scores on the eight questions. Activity intensity ranges were classified as light (I), moderate (II), or heavy (III) according to the two trisectional quantiles of the collected data.

\section{Evaluation of Imagological Data}

Injury to the miaopuapophysial joint induced by foraminoplasty was classified into four grades based on contrasting pre- and postoperative CT imaging: Grade 0, no bony structure injury; Grade I, only part of the ventrolateral bony structure of the superior articular process (SAP) was removed, without involvement of the articular surface or the capsule of the facet joint; Grade II, the lower portion of the foramen was further expanded, and the upper half of the superior articular process was resected, which resulted in partial damage to the ventral capsule of the facet joint and exposure of the articular surface of the inferior articular process; and Grade III, the superior and the inferior articular processes were partially excised (Figure 1).

The location of disc herniation was preoperatively classified as one of four types (central, paramedian (posterolateral), foraminal or far lateral, and migrated herniation) based on preoperative MRI and CT. The grade of disc degeneration was evaluated based on T2-weighted sagittal sequences according to the Pfirrmann criteria. ${ }^{14}$ All of the patients suffered disc degeneration of at least grade II at the operated segment. Therefore, we classified the degree of disc degeneration into four levels (grades 2, 3, 4, and 5) and treated this factor as a categorical variable in the subsequent analysis.

The density of the multifidus and erector spinae muscles was measured by analyzing T1-weighted axial images at the level of the L4/5 disc using the polygon tool in ImageJ software (version 1.52, National Institutes of 


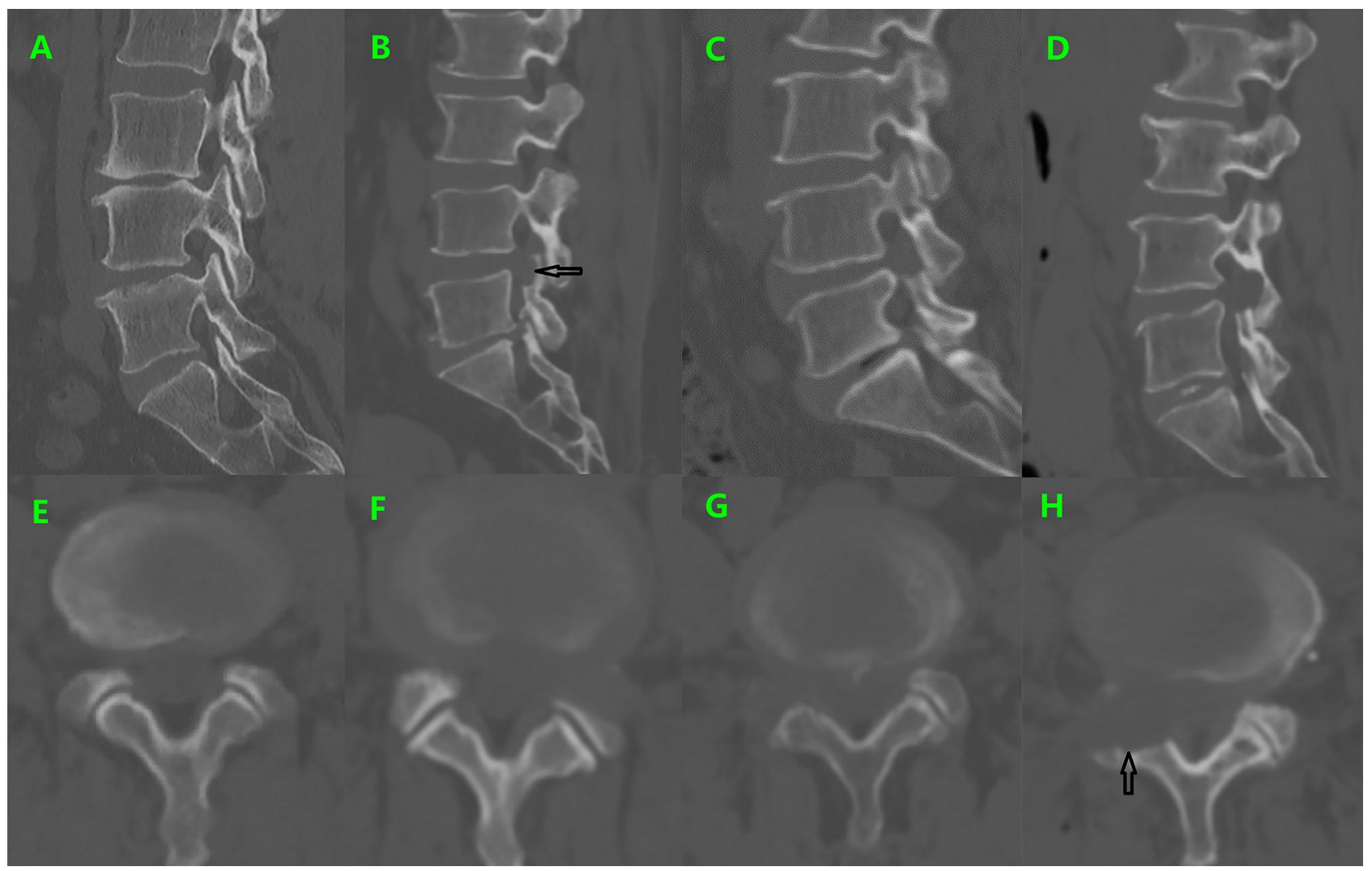

Figure I Postoperative sagittal reconstruction (A-D) and axial (E-H) CT image demonstrates foraminoplasty (black arrowhead) of four grades (0-3) at L4-5 level. L, lumbar.

Health, USA). The result was calculated as the mean of the values obtained for three transaxial slices. The regions of interest (ROI) around the back muscle were outlined with a graphic cursor on the left and right sides. The fascia thoracolumbalis was traced laterally and anteriorly to the dorsal side of the quadratus lumborum, then to the posterior surface of the facet and lamina and finally to the lateral margin of the spinous process. Functional cross-sectional area (FCSA, lean muscle) was estimated according to the method described previously, ${ }^{15}$ and the threshold range was set from 0 to 120 for the discrete grayscale to include only those pixels representing lean paraspinal muscle content in each muscle cross-sectional area (CSA). Areas with a threshold greater than 120 were identified as fatinfiltrated areas. To represent the relative muscle compartment volume in each subject, the FCSA (CSA fat-infiltrated area) of the bilateral muscle compartment was divided by the area of the L4-5 disc (muscle-disc ratio, M/D) (Figure 2). M/D was used to evaluate the lumbar muscularity available to stabilize the spinal column. The Goutallier classification system $(\mathrm{GCS})^{16}$ was used to evaluate the fat infiltration content of the lumbar paraspinal muscles (Figure 3). The GCS was defined as: grade 0 , normal muscle; grade 1 , fatty streaks within the muscle; grade 2, less fat than muscle; grade 3, equal amounts of fat and muscle; and grade 4 , more fat than muscle.

The inferior and superior endplate alignment of L4-5 was used to measure the intervertebral space angle (ISA); the angular intersection located in the dorsal lumbar spine was positive, whereas that in the ventral part was negative (Figure 4A). The sagittal range of motion (sROM) of L4-5 was the absolute value of the hyperflexion (B) - the hyperextension (C) angle (Figure 4). The disc height index (DHI) was calculated from lateral radiographs using a previously described method (Figure 5A) ${ }^{17}$ Static curve characteristics, including the Cobb angle in the coronal plane and lumbar lordosis (LL), were estimated on the anteroposterior and lateral radiographs. The Cobb angle was the included angle between the extending line of the superior endplate of the cranial vertebra (the vertebra with the greatest inclination angle above the apex of the scoliotic curve) and the inferior endplate of the caudal vertebra (vertebra with greatest declination angle below the apex of the scoliotic curve) (Figure 5B). According to Kim et al, ${ }^{18}$ a Cobb 


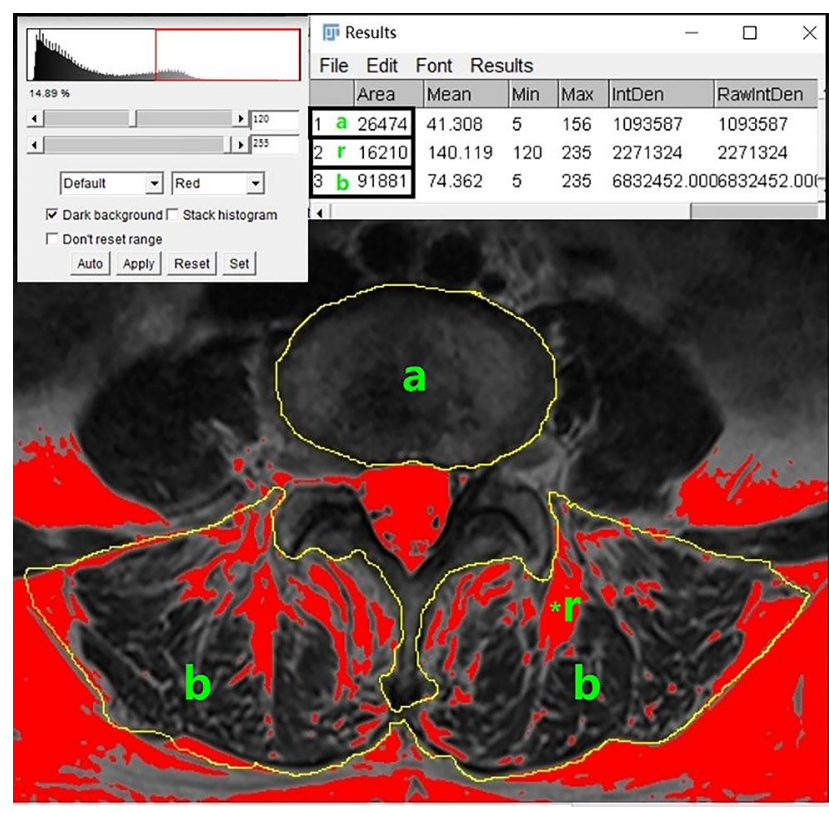

Figure 2 Axial TI-weighted MR image demonstrating measurement of the functional muscle-disc ratio by creating ROls with Imagej software. (A), L4/5 disc area. (B), cross-sectional area of paravertebral muscle. *r, bright pixels of fat tissue (with threshold >120) in ROls are colored in red. M/D was defined as (b-r)/a.

Abbreviations: ROI, regions of interest; $M / D$, muscle-disc ratio; L, lumbar.

angle ranging from 0 to 10 degrees was defined as curvature progression, an angle of between 11 and 30 degrees was defined as mild scoliotic curvature, an angle of 30 to 50 degrees indicated moderate curvature, and an angle greater than 50 degrees indicated severe curvature. The angles of all subjects in this research were no greater than 30 degrees, and we defined this factor as a classification variable according to the presence or absence of scoliosis. LL was defined as the angle between the vertical line of the upper edge of L1 and the vertical line of the upper endplate of S1 (Figure 5C). All angle measurements were performed using the measuring tools included in Centricity RIS/PACS CE.

In an attempt to prevent interobserver variability, same observer, who was blinded to patient identifiers, performed all measurements three times.

\section{Statistical Analysis}

Clinical and radiological parameters during the hospitalization and follow-up periods of the patients were assessed, and univariate analysis was performed to identify the potential risk factors for overall recurrence rate with the Kaplan-Meier method. Multiple regression analysis using the Cox proportional hazards model was performed with variables for which $\mathrm{p}<0.1$ in the univariate analysis to determine independent risk factors for rLDH. Continuous variables are described as the mean values with standard deviations [SD].

All statistical analyses were performed using $\mathrm{R}$ programming language, version 3.6.0 (R Foundation), and p-values of $<0.05$ were considered statistically significant.

\section{Results}

A total of 654 patients were enrolled in this study, and 46 (7.0\%) developed rLDH after primary surgery. The demographic and clinical characteristics of patients in the two groups are shown in Table 1.

Univariate analysis was performed for the effects of clinical parameters (age, gender, BMI, duration of symptoms, hypertension history, diabetes mellitus history, postoperative smoking and alcohol use, and physical load intensity), and radiological parameters (foraminoplasty grade, herniation location, grade of disc degeneration, muscle-disc ratio, percentage of fat infiltration, sROM, DHI, intervertebral space angle, scoliosis, and LL) on recurrence. Of these factors, old age, high BMI, diabetes mellitus history, smoking, large physical load intensity, moderate disc degeneration, small muscle-disc ratio, more fat infiltration, large sROM, scoliosis, small DHI, small ISA, and small LL were considered potential risk factors $(\mathrm{p}<0.10)$ for predicting rLDH (Table 1). Continuous variables (age, BMI, SPHQ, DHI, ISA, LL, muscle-disc ratio, and SROM) were classified as categorical variables according to trisectional quantiles or medians. The Kaplan-Meier curves for recurrence-free

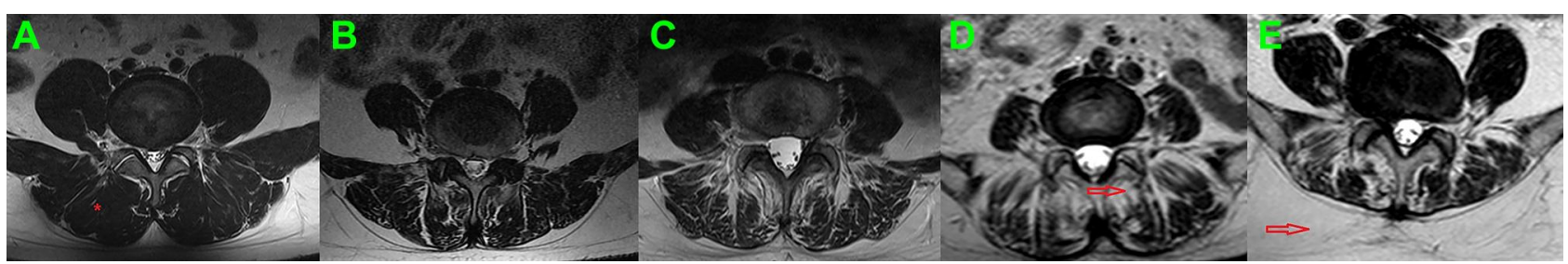

Figure 3 Goutallier grades 0 to 4 on TIW axial MRIs obtained at the L4-5 level are represented in Figures (A-E). The asterisk (A) indicates normal multifidus muscle signal, and the arrowhead indicates the high-signal fat located between the muscle and adjacent erector spinae (D), or in the subcutaneous area (E). The infiltration of fatty tissue gradually increases as the grade intensifies. Abbreviation: L, lumbar. 


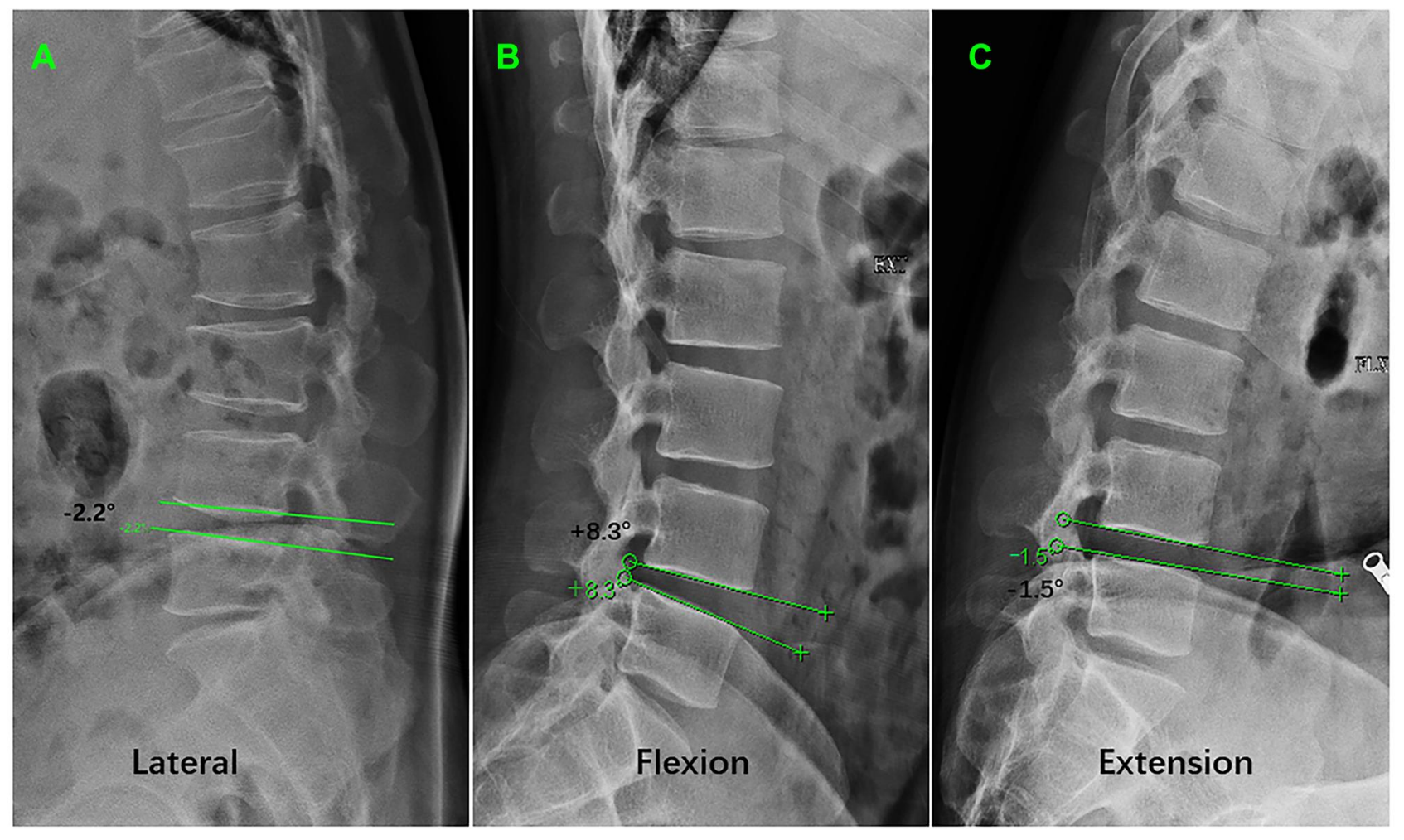

Figure 4 Method of measuring ISA (A) and SROM (B and C) using the superior and inferior endplate of L4 -L5 on the lateral view. (A) positive ISA was defined by ventrally located angular intersection of lumbar spine, and a negative angle was defined by dorsally located angular intersection. The ISA was $-2.2^{\circ}$ in a case of recurrent LDH (L4-5). (B and C) The sagittal range of motion (sROM) of L4 -L5 was defined as the absolute value of extension (B) - flexion (C) angles. The sROM at L4-5 was $9.8^{\circ}$ in another patient.

Abbreviations: ISA, intervertebral space angle; sROM, sagittal range of motion; L, lumbar.

survival rates according to the significant risk factors are shown in Figures 6 and 7.

These potential risk factors were analyzed using a multivariate Cox regression model. Patients with high BMI, large physical load intensity, moderate disc degeneration, small muscle-disc ratio, more fat infiltration, large sROM, small ISA, or small LL were more likely to relapse (Table 2, Figure 8). No significant association was identified between $\mathrm{rLDH}$ and any other potential risk factors (age, duration of symptoms, diabetes mellitus history, smoking, or scoliosis). A further breakdown analysis was also performed based on the eight subitems in SPHQ, four of which (B, D, E, and F) were associated with rLDH (Table 3).

\section{Discussion}

The recurrence rate of rLDH at the L4-5 level after successful PETD between October 2013 and January 2020 in our department was $7.0 \%$. The literature reported age, gender, BMI, smoking and alcohol use, diabetes, disc degeneration, herniation type, and herniation location as risk factors for rLDH after PETD. ${ }^{8,19}$ However, $\mathrm{Kim}^{7}$ and
Yin et al, ${ }^{4}$ found that gender, smoking, and level and type of herniation were not associated with LDH recurrence after PELD. Many clinical and biomechanical parameters are involved, which makes it difficult to establish a consensus and draw reliable conclusions of risk factors for rLDH.

The present study found that the incidence of rLDH was higher in discs with moderate degeneration (grade III) than discs with mild degeneration (grade II), which is consistent with previous correlational research. ${ }^{20}$ Severely degenerated discs (grades IV and V) also experienced infrequent rLDH in the present study. Relevant cadaveric and clinical studies demonstrated that the range of segmental motion in degenerated and collapsed discs decreased in proportion to the degree of degeneration. ${ }^{21}$ Hasegawa et al proposed that moderately degenerated segments with preserved disc height had a latent instability compared to severely degenerated, collapsed discs, and Kirkaldy-Willis et al further divided disc degeneration into three phases: (1) temporary dysfunction; (2) instability; and (3) restabilization., ${ }^{9,22}$ These data suggest that the biomechanical stability of collapsed discs explained the 

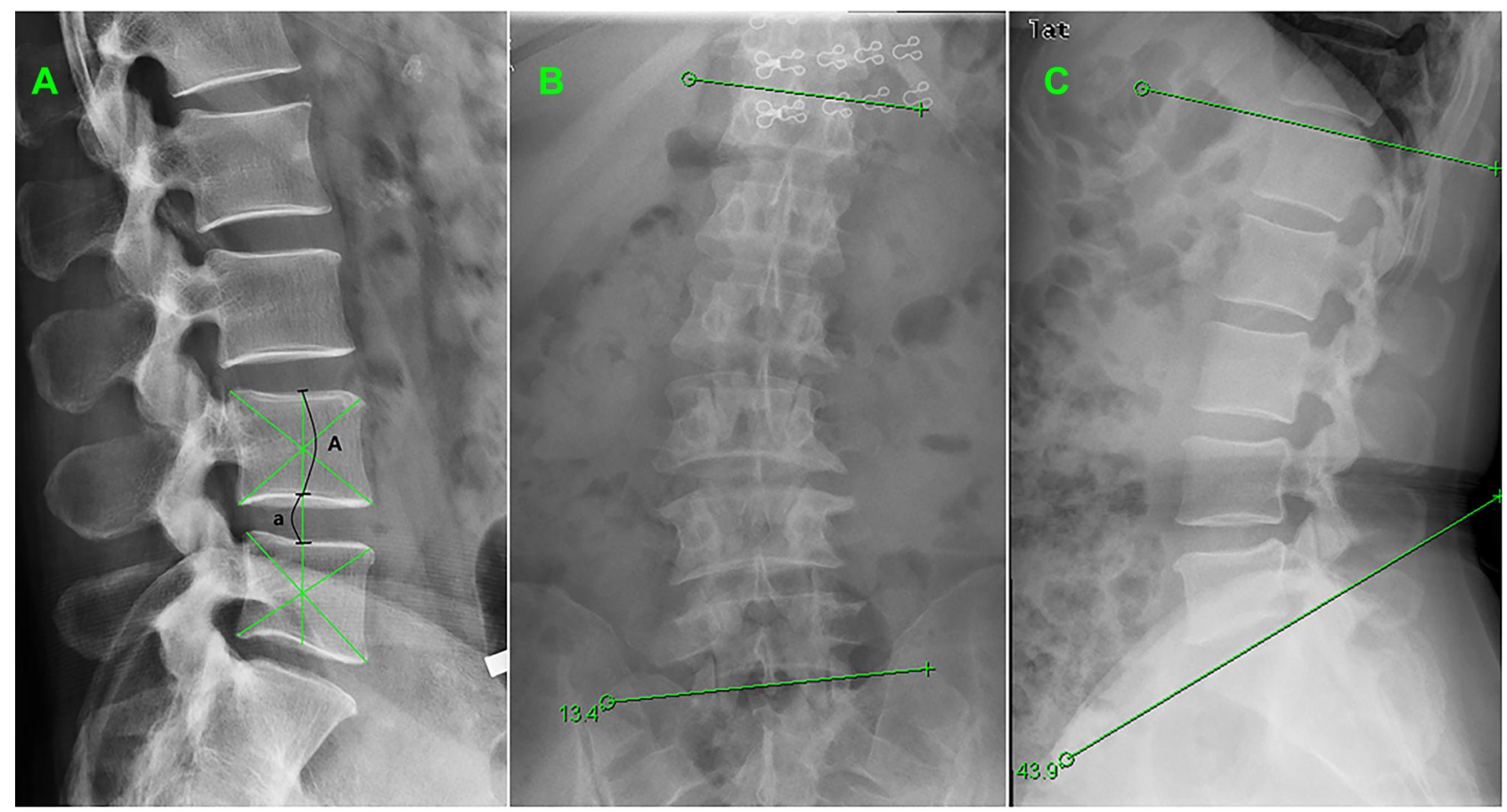

Figure 5 Radiographic measurement of DHI, Cobb angle and LL. (A), Mid-vertebral line (the line passing through the intersection of 2 diagonals of L4 and L5) was used to measure the heights of vertebral body and disc. $\mathrm{DHI}=\mathrm{a} / \mathrm{A} .(\mathbf{B})$, Cobb angle was defined as the crossing angle between the extension line of the upper and inferior edge of the apex vertebrae. (C), LL was the angle between the extension line of the upper edge of LI and the extension line of the upper endplate of SI.

Abbreviations: DHI, disc height index; LL, lumbar lordosis angle; L, lumbar; S, sacrum.

low incidence of rLDH in severely degenerated discs. Limited content generally remained in severely degenerated discs. These discs had less height after removal of the protrusion, and much of the content was replaced by tough collagen fibers or ossified tissues, which suggests that lower residential disc height may be an indicator of the stabilization of the intervertebral space and reduced chance of rLDH, but no statistically significant association was found ( $\mathrm{P}=0.077$, Figure 8$)$.

Most investigations found that rLDH was more likely to occur with advancing age. ${ }^{7}$ Yao et $\mathrm{al}^{8}$ in 2017 and $\mathrm{Wu}$ et $\mathrm{al}^{23}$ in 2018 also reported that older age was a predisposing factor for recurrence. This finding is reasonable because older discs are typically characterized by increased degeneration and have a tendency to deform more easily. Annular collagen changes and annular tears were present in most degenerated discs. Therefore, the healing processes of the outer lamellae after surgical management may not be sufficient to effectively reconstitute the external annulus. ${ }^{24,25}$ Although our univariate analysis suggested an obvious relationship between increased age and $\mathrm{rLDH}$, multivariate Cox regression analysis showed no significance of this factor. This difference may be due to selection bias. The aging population generally engages in relatively less physical activity than the middle-aged population. Members of the latter group generally have degenerative changes in intervertebral discs but simultaneously experience labor intensity that is similar to the younger age group. ${ }^{12}$

In ergonomic epidemiology, evaluation based on the eight questions listed in the SPHQ is a valid research approach for daily physical workload intensity and level, duration, and frequency of exposure in musculoskeletal disorders (MSDs), including spinal diseases. ${ }^{13,26}$ None of the subjects in the current study engaged in excessive physical activity after PETD. No subject in this group had a total SPHQ score greater than 26 points. We performed a separate subgroup analysis of each item in the questionnaire and found an apparent association between recurrent disc herniation and increased frequency of manual lifting of $10-\mathrm{kg}$ loads in daily life. An increased proportion of workday spent twisting the trunk, excessive bent/twisted work posture, and spending too much time sitting were also primary factors that influenced rLDH occurrence (Table 1). Although the activities evaluated in this study differed slightly from the factors used in other LDH-related occupational research, similar results were obtained. ${ }^{27}$ We postulate that long-term cumulative 
Table I The Demographic and Clinical Characteristics of Patients in Two Groups and Results of Univariate Analysis of Risk Factors Predicting Recurrence After Percutaneous Endoscopic Transforaminal Discectomy

\begin{tabular}{|c|c|c|c|}
\hline Risk Factors & $\begin{array}{l}\text { R Group } \\
(n=46)\end{array}$ & $\begin{array}{l}\text { N Group } \\
(n=608)\end{array}$ & $P$ value \\
\hline Follow-up (months) & $27.6 \pm 8.2$ & $29.7 \pm 11.2$ & I \\
\hline Age $(Y / M / O)^{a}$ & $55.4 \pm 12.2$ & $44.9 \pm 12.8$ & $<0.001$ \\
\hline Gender (male/female) & $22 / 24$ & $333 / 275$ & 0.34 \\
\hline $\operatorname{BMl}\left(\mathrm{Kg} / \mathrm{m}^{2}\right)$ & $24.70 \pm 1.67$ & $23.17 \pm 1.58$ & $<0.001$ \\
\hline Symptom duration (months) & $7.1 \pm 2.1$ & $6.3 \pm 2.5$ & 0.55 \\
\hline Hypertension (yes/no) & $10 / 36$ & $98 / 510$ & 0.51 \\
\hline Diabetes mellitus (yes/no) & $11 / 35$ & $76 / 532$ & $<0.05$ \\
\hline Smoking (yes/no) & $14 / 32$ & $91 / 517$ & $<0.01$ \\
\hline Alcohol use (yes/no) & $13 / 33$ & $116 / 492$ & 0.12 \\
\hline Physical load intensity (points) $(\mathrm{I} / \mathrm{I} / \mathrm{I} / \mathrm{II})^{\mathrm{a}}$ & $18.8 \pm 3.8$ & $17.7 \pm 2.6$ & $<0.05$ \\
\hline Foraminoplasty grade $(0 / I / I I / I I I)$ & $6 / 13 / 15 / 12$ & $159 / 139 / 168 / 142$ & 0.21 \\
\hline Herniation location (C/P/F/M') & $13 / 13 / 13 / 7$ & $165 / 212 / 155 / 76$ & 0.93 \\
\hline Disc degeneration $(2 / 3 / 4 / 5)$ & $7 / 27 / 11 / 1$ & $222 / 96 / 154 / 136$ & $<0.001$ \\
\hline Muscle-disc ratio $(\mathrm{L} / \mathrm{S})^{\mathrm{a}}$ & $2.19 \pm 0.17$ & $2.38 \pm 0.18$ & $<0.001$ \\
\hline Fatty infiltration $(0 / 1 / 2 / 3 / 4)$ & $0 / 9 / 14 / 15 / 8$ & $4 / 224 / 253 /|2| / 6$ & $<0.001$ \\
\hline Sagittal range of motion $\left({ }^{\circ}\right)(\mathrm{L} / \mathrm{S})^{\mathrm{a}}$ & $8.6 \pm 3.0$ & $7.5 \pm 1.5$ & $<0.05$ \\
\hline Disc height index (L/S) ${ }^{a}$ & $0.20 \pm 0.04$ & $0.24 \pm 0.07$ & $<0.01$ \\
\hline Intervertebral space angle $\left({ }^{\circ}\right)(\mathrm{L} / \mathrm{S})^{\mathrm{a}}$ & $3.2 \pm 3.2$ & $6.1 \pm 1.7$ & $<0.001$ \\
\hline Scoliosis (yes/no) & $32 / 14$ & $533 / 75$ & $<0.001$ \\
\hline Lumbar lordosis $\left({ }^{\circ}\right)(\mathrm{L} / \mathrm{S})^{a}$ & $31.3 \pm 3.5$ & $38.3 \pm 5.6$ & $<0.001$ \\
\hline
\end{tabular}

Note: ${ }^{a}$ Variable was classified as categorical variables according to tri-sectional quantiles or median.

Abbreviations: BMI, body mass index; C, centra; P, paramedian (posterolateral); F, foraminal or far lateral; M', migrated; Y, young; M, moderate; O, old; L, large; S, small.

postures that involve extreme forward bending or a twisted position and sedentary activity play etiological roles in increased risk of disc protrusion recurrence. These findings indicated that the residual nucleus materials in degenerated discs with preserved intervertebral height were, to a certain extent, susceptible to relapse when subjected to persistent mechanical load.

Of the radiological parameters investigated in the present study, we defined the inferior and superior endplate alignment with dorsally located angular intersection to be positive and the ventrally located intersection to be negative. A numerically smaller (or dorsal-opening) intervertebral space angle and large sagittal range of motion were important risk factors for rLDH. Biomechanically, a smaller or negative ISA indicates that the posterior intervertebral space is wide enough, and the residual disc tissue had more opportunity to move backward from its original position than in cases with forward-opening ISA when pressed by the upper and lower endplates and may be extruded from annulus fibrous rupture. This movement may also explain the association between bending posture and rLDH to some extent. Therefore, patients were generally advised to maintain a straight back posture in daily life to maintain a positive ISA and prevent the nucleus pulposus from moving backwards. However, high sROM caused stress on the corresponding disc and indicated insufficient intervertebral space stability, both of which affect the development of $\mathrm{rLDH}$ or primary $\mathrm{LDH}^{17}$ However, the intrinsic limitation of our method was that ROM was checked only from the sagittal view, and multidirectional measurement of the L4-5 segment, which was not a routine part of the examination prior to surgery in our department, would be more desirable. Therefore, compensatory analysis of the static morphology of the spine, including lumbar lordosis angle and scoliosis, was performed. The presence of spinal malformations, such as scoliosis, is associated with vertebral instability and asymmetric spinal biomechanical loading, which may result in the subsequent accelerated degeneration of adjacent intervertebral discs and altered distribution of intervertebral disc extrusions. ${ }^{28,29}$ Furthermore, the distal segments are more susceptible to this mechanism compared to the deformed segments. ${ }^{30}$ As an important spinal component of physiological curvature of the lumbar spine, LL affects the intervertebral mechanical stress at the L4-5 segment. Long-term LDH may lead to a straightening of the 

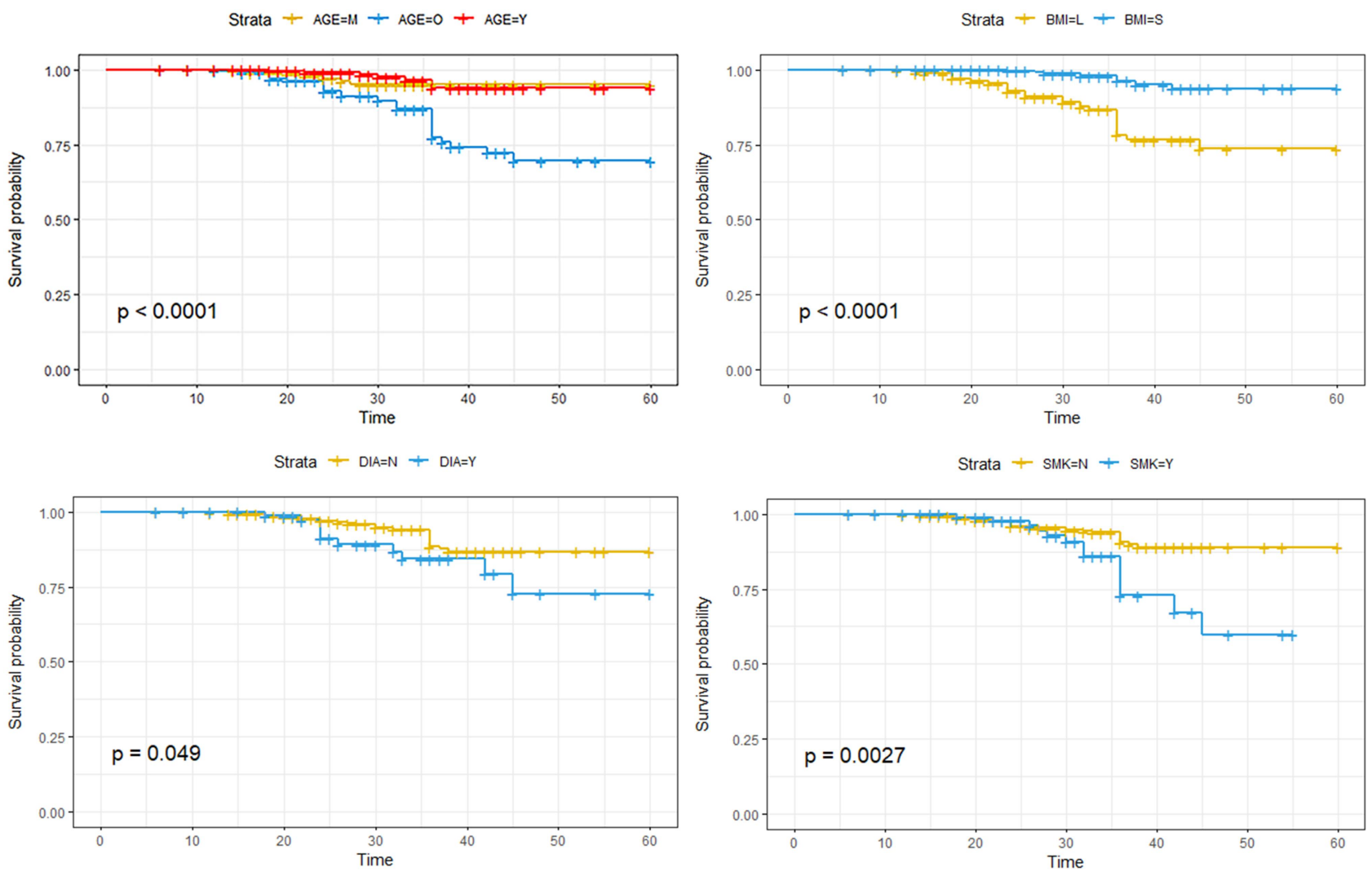

Figure 6 Kaplan-Meier curves indicating recurrence-free survival rate according to the identified significant risk factors for rLDH. Shown here are the potential risk factors in demographic characteristics.

Abbreviations: BMI, body mass index; DIA, diabetes; SMK, smoking; M, moderate; O, old; Y (in AGE), young; L, large; S, small; Y, yes; N, no; rLDH, recurrent lumbar disc herniation.

physiological curvature of the lumbar spine and unidirectionally increases the forward spinal pressure, which disrupts the equilibrium of shear force, weakens intervertebral space stability, and increases the retrusive trend of the residual intervertebral disc. The recurrence of LDH at the L4-5 level in our study was significantly associated with smaller lumbar lordosis angle. Chang et al demonstrated that patients with spinal scoliosis were at higher risk of recurrence of symptoms after surgery. ${ }^{31}$ The present study found no significant relationship between recurrent LDH at the L4/5 level and scoliosis, and this finding is inconsistent with previous studies. The difference in the results may be because the current study found no severe spinal malformation, and the distal spinal section instead experienced a sagittal imbalance that was not obvious. Although it may be hypothesized that severe spinal curvature abnormalities are related to more evident vertebral biomechanical loading alterations, further research is necessary to assess whether more serious scoliosis is associated with a higher risk of developing $\mathrm{rLDH}$.
The lumbar zygapophyseal joints serve important functions in lumbar motion in guiding the movement of the spine and resisting compression. ${ }^{32,33}$ Some scholars demonstrated that the destruction of lumbar facet joints disrupts the motion of responsible segments to resist torsional load, which increases the range of lumbar motion and intervertebral instability and finally leads to or accelerates the degeneration of intervertebral discs. ${ }^{34,35}$ Foraminoplasty to enlarge the intervertebral foramen near the facet joint by cutting the anterior aspect of the superior articular process (SAP) is generally recommended in PETD. ${ }^{36,37} \mathrm{Li}$ et $\mathrm{al}^{38}$ investigated the pathoanatomy and changes in the stability of cadaveric functional spinal units (FSU) and found that overenlarged posterolateral foraminoplasty at L4-5 with impairment of the articular surface and ventral capsule of the facet joint significantly decreased, but did not destroy, the stability of lateral bending, and limited posterolateral foraminoplasty without impairment to the articular surface and capsule of the zygapophyseal joint did not impair lumbar stability. Qiao et al observed a recurrence rate of $6.25 \%$ 

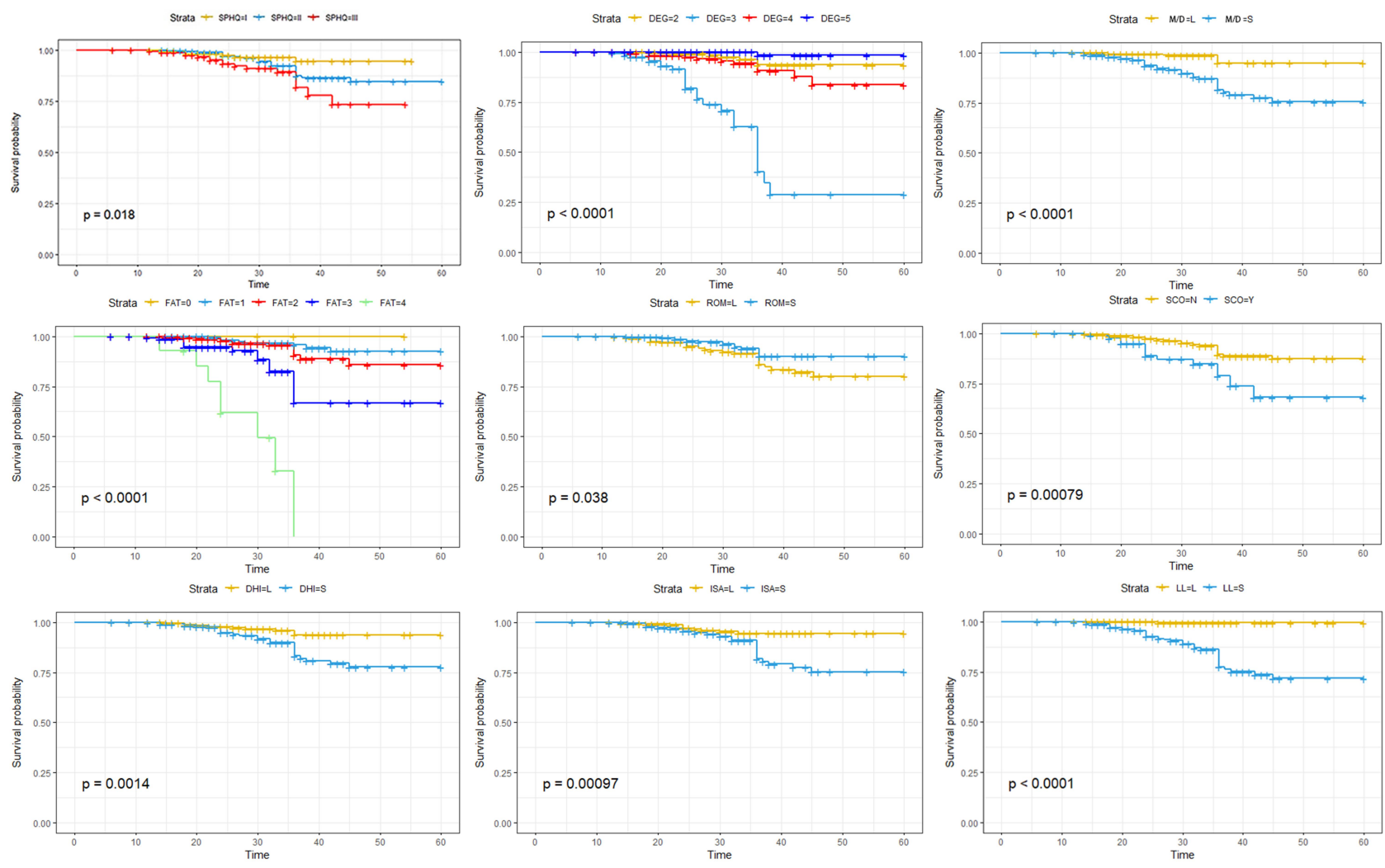

Figure 7 Kaplan-Meier curves for recurrence-free survival rate based on potential risk factors of rLDH related to biomechanical characteristics. Abbreviations: SPHQ, Stockholm Public Health Questionnaire; DEG, disc degeneration; M/D, muscle-disc ratio; FAT, fat infiltration of paravertebral muscle; ROM, sagittal range of motion; SCO, scoliosis; DHI, disc height index; ISA, intervertebral space angle; LL, lumbar lordosis angle. L, large; S, small; Y, yes; N, no.

at two-year follow-up after percutaneous endoscopic lumbar discectomy foraminoplasty (PELDF). This rate was higher than the group with no facet joint injury, which suggests that foraminoplasty destroys the stability of the zygapophyseal joint of the lumbar vertebrae by breaking important structures, ie, the joint capsule and joint surface, and it was a risk factor for LDH recurrence. ${ }^{39}$ However, Ruan et al $(2017)^{40}$ reported that no biomechanical or clinical difference was associated with foraminoplasty in different amplitudes, and foraminoplasty had no obvious effect on the stress distribution of the joints based on a 3D finite element model of L4-5 segments. The results of the present study showed that foraminoplasty grade was not significantly associated with rLDH occurrence. We speculate that the biomechanical changes induced by zygapophyseal joint destruction are gradual and that a longer follow-up period (at least 5 years) is required to fully determine the potential risk of foraminoplasty for rLDH.

Previous scholars considered that extensive degeneration, weakness, and increased fat infiltration of back extensor muscles, were important causative factors in the pathogenesis of degenerative lumbar diseases (DDD),${ }^{41-43}$ and the pathogenic effect of muscle disorder in rLDH was also confirmed in the present investigation. Atrophy of the paraspinal muscles that provide dynamic balance to the lumbar spine may result in instability of the spine and exacerbate disc and facet degeneration. ${ }^{44}$ Kalichman et $\mathrm{al}^{45}$ found that spinal degeneration features, including disc narrowing and facet joint osteoarthritis (FJOA), were negatively associated with the density of the multifidus erector spinae. Realistically, the relationship between muscle atrophy and DDD may be explained as a vicious cycle. Previous studies demonstrated that older and overweight individuals had lower paraspinal muscle density and FCSA than younger and lower-weight individuals, ${ }^{45,46}$ which suggests that changes in FCSA and the density of the paraspinal muscles are associated with variables, such as advanced age and overweight. Therefore, therapeutic-intensity paraspinal muscle function exercise is generally advised for patients with these characteristics to reduce the risk of accelerated disc degeneration and rLDH by promoting the balance of FSU. 
Table 2 Results of Multivariate Analysis of Potential Risk Factors Predicting Recurrence After Percutaneous Endoscopic Transforaminal Discectomy

\begin{tabular}{|c|c|c|c|c|}
\hline \multicolumn{2}{|l|}{ Risk Factors } & $\begin{array}{l}\text { Hazard } \\
\text { Ratio }\end{array}$ & $95 \% \mathrm{Cl}$ & $P$ value \\
\hline \multicolumn{2}{|l|}{ Age } & 1.011 & $0.966-1.058$ & 0.64 \\
\hline \multicolumn{2}{|l|}{ BMI } & 1.391 & $|.092-| .77 \mid$ & $<0.01$ \\
\hline \multicolumn{2}{|l|}{ Diabetes mellitus } & 1.864 & $0.791-4.392$ & 0.15 \\
\hline \multicolumn{2}{|l|}{ Smoking } & 1.267 & $0.565-2.843$ & 0.57 \\
\hline \multicolumn{2}{|c|}{ Physical load intensity } & 1.154 & $1.041-1.279$ & $<0.01$ \\
\hline \multirow[t]{3}{*}{ Disc degeneration } & $3^{\#}$ & 4.964 & $1.292-19.068$ & $<0.05$ \\
\hline & $4^{\#}$ & 0.606 & $0.110-3.34 \mid$ & 0.57 \\
\hline & $5^{\#}$ & 0.606 & $0.009-1.847$ & 0.13 \\
\hline \multicolumn{2}{|l|}{ Muscle-disc ratio } & 0.0429 & $0.002-0.753$ & $<0.05$ \\
\hline \multicolumn{2}{|l|}{ Fatty infiltration } & 1.996 & $1.204-3.308$ & $<0.01$ \\
\hline \multicolumn{2}{|c|}{ Sagittal range of motion } & 1.159 & $1.004-1.338$ & $<0.05$ \\
\hline \multicolumn{2}{|c|}{ Disc height index } & 0.131 & $0.000-2.849$ & 0.0775 \\
\hline \multicolumn{2}{|c|}{ Intervertebral space angle } & 0.863 & $0.747-0.997$ & $<0.05$ \\
\hline \multicolumn{2}{|c|}{ Scoliosis } & 0.6999 & $0.338-1.450$ & 0.33 \\
\hline \multicolumn{2}{|l|}{ Lumbar lordosis } & 0.8777 & $0.790-0.975$ & $<0.05$ \\
\hline
\end{tabular}

Note: ${ }^{\#}$ Compared to degeneration grade 2 .

Abbreviations: $\mathrm{Cl}$, confidence interval; $\mathrm{BMI}$, body mass index.

Kim et $\mathrm{al}^{7}$ conducted a study that included 361 participants with reoccurrence of disc herniation among 4861 patients who underwent PELD and detected no difference in the proportion of men and women, which is consistent with our findings. Cinotti et $\mathrm{al}^{47}$ and Suk et $\mathrm{al}^{11}$ investigated the association between gender and rLDH and reported that males with significant degenerative disc disease were more likely than females to experience recurrent symptoms after percutaneous endoscopic lumbar surgery. The discrepancy in the effects of estrogen and androgen levels may underlie the gender differences in $\mathrm{rLDH}$, but the difference may be negligible, and relevant in-depth investigations are rare in the literature.

Kara et $\mathrm{al}^{48}$ found no significant association between rLDH and BMI. However, patients with higher BMI presented a higher recurrence rate in the present study, which suggests that shorter height with more weight increased the load on the disc. Studies also consistently demonstrated that consequent periodic increases in intradiscal pressure led to higher shear strain on the posterolateral part of the annulus fibrosus and an aggravated burden on the disc. ${ }^{7}$ When combined with other factors, increased BMI may significantly influence the biomechanical characteristics and morphology of the disc, especially in the case of degenerated and postoperative discs.
Wang et $\mathrm{al}^{19}$ and Huang et $\mathrm{al}^{49}$ demonstrated that patients with diabetes mellitus, smoking, and alcohol use had increased risk of rLDH occurrence and found that proteoglycans, which band at lower buoyant density and are induced by hyperglycemia or nicotine from cigarette smoking, affected disc annulus nutrition and oxygenation and inhibited the healing process, especially during early rehabilitation after surgery. However, the current study failed to verify that smoking status or diabetes was a significant risk $^{49}$ factor for rLDH. Most patients made some lifestyle changes, such as reduced smoking, diet control, and reduced alcohol intake, following surgery. The pathological changes mentioned above, which may have exerted a slight influence originally, were reversed to some extent so that the reparative processes of the operative disc would proceed normally. Whether the above factors have synergistic or amplifying effects in the healing process after micro-operation must be further investigated. Therefore, long-term and high-level evidence-based prospective studies are needed to determine the effects of these factors on recurrence following disc surgery.

In contrast to the findings reported in other research, we found that the initial type of disc herniation at the recurrent segment showed no obvious difference in patients with and without rLDH. Notably, scholars have not always reported consistent results. Kim et $\mathrm{al}^{12}$ reported that patients with central and far lateral herniation were less likely to experience relapse compared to patients with paracentral disc herniation, but Yao et $\mathrm{al}^{8}$ reached the opposite conclusion that patients with central herniation were more likely to experience rLDH. The latter investigators believed that the centrally located protrusion was not easily accessible for complete excision because of the difficulty of placing the working channel near the herniated content. A more vertical approaching trajectory may be an excellent choice when placing the working channel inside during the treatment of paracentral and far lateral disc herniation. In contrast, a more horizontal trajectory angle is a priority in cases of central herniation, and the contralateral portion may also be accessible (Figure 9). Other researchers also demonstrated that migrated protrusion was a potential risk factor for rLDH after PELD because of the difficulty in removing the hidden fragments beyond the working area under limited endoscopic view. ${ }^{7}$ A deliberate surgical strategy may be developed with the aid of thorough 


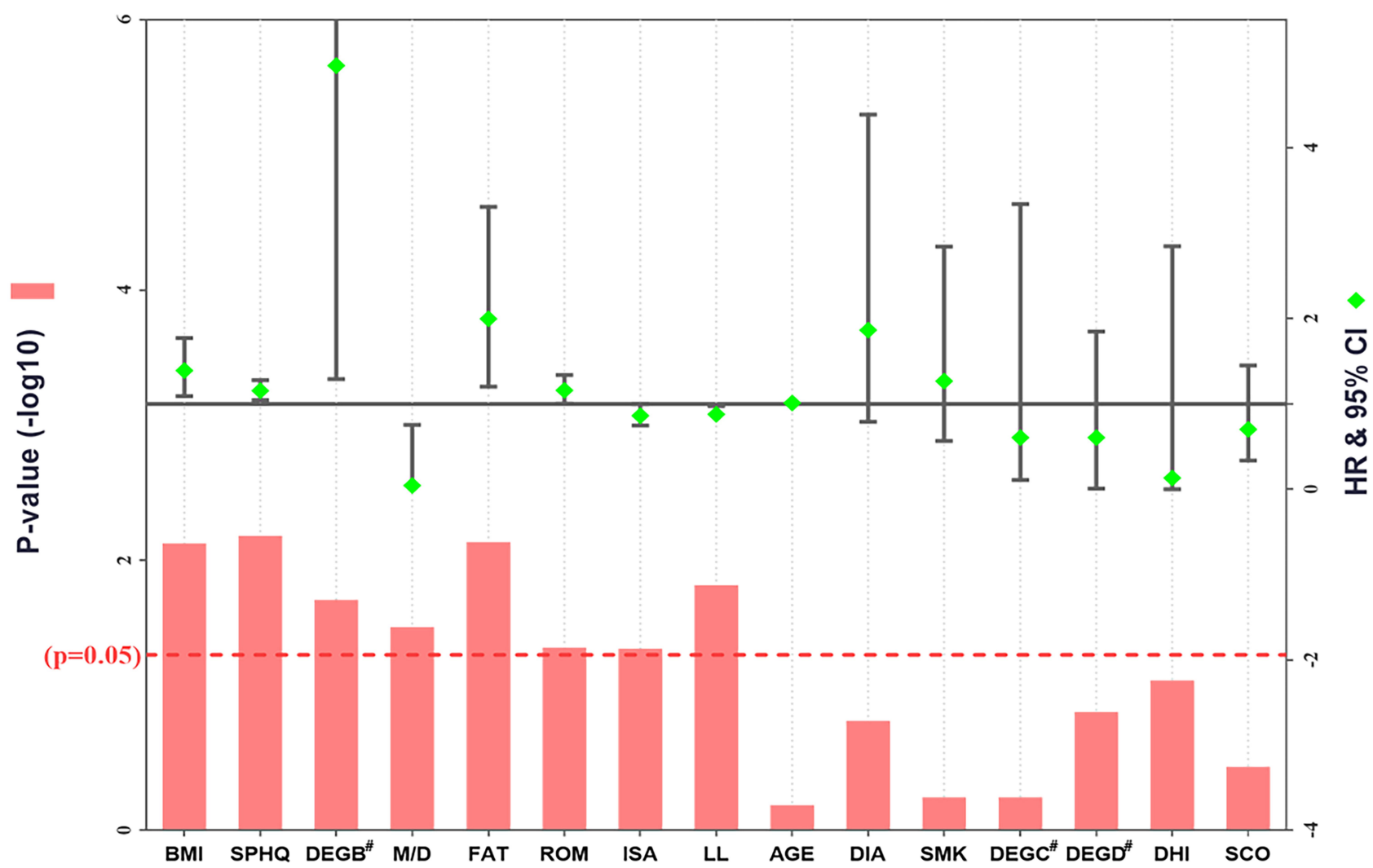

Figure 8 Results of multiple regression analysis using the Cox proportional hazards model. ${ }^{\#}$ Compared to DEG A (grade 2); p < 0.05, column diagram past red dashed line. Abbreviations: BMI, body mass index; SPHQ, Stockholm Public Health Questionnaire; M/D, muscle-disc ratio; FAT, fat infiltration of paravertebral muscle; ROM, sagittal range of motion; ISA, intervertebral space angle; LL, lumbar lordosis angle; DIA, diabetes; SMK, smoking; DHI, disc height index; SCO, scoliosis; DEG (B/C/D), disc degeneration grade (3/4/5); HR, hazard ratio; $\mathrm{Cl}$, confidence interval.

preoperative examination, including $\mathrm{X}$-ray, $\mathrm{CT}$, and MRI. Postoperative review using MRI was also performed in our study to ensure that the herniated disc was entirely removed. Normally, experienced surgeons reduce the iatrogenic failure of endoscopic surgery referenced above.

Table 3 The Result of Further Breakdown Analysis for Eight Items in the Stockholm Public Health Questionnaire Using the Cox Proportional Hazards Model

\begin{tabular}{|l|l|l|l|}
\hline Subitems & HR & 95\% CI & P value \\
\hline B. Sitting work posture & 2.104 & $1.230-3.598$ & 0.007 \\
D. Trunk bent & 1.743 & $1.064-2.854$ & 0.027 \\
E. Bent/twisted work posture & 1.818 & $1.179-2.803$ & 0.007 \\
F. Manual lifting of loads I0 kg & 2.099 & $1.303-3.38 \mathrm{I}$ & 0.002 \\
A. General physical activity & 1.158 & $0.895-2.576$ & 0.122 \\
C. Hands above shoulder level & 1.235 & $0.790-1.931$ & 0.354 \\
E. Repetitive movements & $0.98 \mathrm{I}$ & $0.674-1.429$ & 0.922 \\
F. Physical exercise/sports & 1.147 & $0.677-1.943$ & $0.61 \mathrm{I}$ \\
\hline
\end{tabular}

Abbreviations: $\mathrm{HR}$, hazard ratio; $\mathrm{Cl}$, confidence interval.
The postoperative scar tissue at the original position of the resected disc has biomechanical characteristics that are not the same as the elastic annulus fibrosus, and it may be relatively fragile and more susceptible to mechanical stress. Previous literature that analyzed risk factors for rLDH after PELD primarily focused on the surgeon level, surgical approaches, demographic or lifestyle characteristics, such as gender, age, smoking, BMI, and trauma history. ${ }^{7,23,50}$ Other studies examined the association between $\mathrm{rLDH}$ and anatomical factors, including disc degeneration, history of discectomy, herniated disc level, disc height, segmental dynamic motion, and disc location. However, fewer investigators performed comprehensive analyses of these factors, and the cumulative effect of time was not always considered. Intensive studies of the biomechanical characteristics of the operative segment are limited. The present research performed multivariate Cox regression analysis on the relationship to $\mathrm{rLDH}$ of the factors mentioned above. Foraminoplasty foraminotomy grade, fatty infiltration degree and content of paravertebral muscle, which act as significant biomechanical 


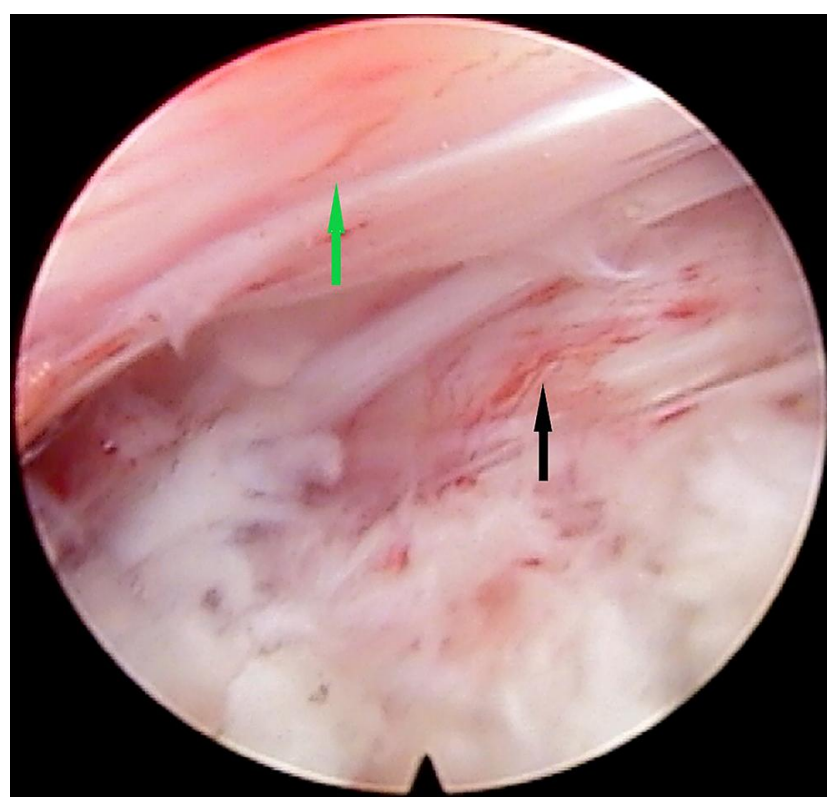

Figure 9 The operation field under endoscopy in PETD. The contralateral L5 nerve root (black arrowhead) is accessible and can be easily decompressed. The green arrowhead demonstrates the dural sac.

Abbreviation: PETD, percutaneous endoscopic transforaminal discectomy.

characteristics, were also evaluated. Based on our research, we present many recommendations to help reduce the recurrence rate. For the surgeons, 1) it is important to select appropriate patients with fewer risk factors before surgery; and 2) complete preoperative examination is needed to ensure that the pathological disc may be entirely removed. For patients, 1) lifestyle improvement may work, such as losing weight, quitting smoking, and positively controlling blood sugar; 2) trying to tighten the stomach and lift the chest to keep the spine straight in daily life, and reduce the frequency or intensity of posture (shown in Table 3), which bends the physiological curve of spine; and 3) light exercise for the muscle group of the back and waist at least 3 months after surgery, when the scar is strong enough.

\section{Limitations}

This retrospective nonrandomized case-control study involved patients with intrinsic defects, and the number of subjects with diagnosed $\mathrm{rLDH}$ in the current research was relatively small. Many patients who experience recurrent disc herniation without symptoms of radiating pain may not undergo imaging examination. Therefore, systematic radiological follow-up for all eligible patients may be more reasonable to ensure that asymptomatic recurrent disc herniation is identified. Parameters of spinal kyphosis (TK), the C7 plumb line (PL), and the sagittal vertical axis (SVA) from C7 PL were associated with the biomechanical characteristics of discs. ${ }^{51}$ Regrettably, it was difficult to perform further research using these data because of the lack of related preoperative examination. This lack of data is an inherent limitation of retrospective studies. Another matter that should be mentioned is the technical limitation imposed by difficulties in defining the muscle boundaries. Therefore, we were unable to perform individual analyses of the multifidus, longissimus, and iliocostalis muscles. Some of the investigated factors may affect the characteristics of the original disc with increased exposure time. Further high-quality observational studies and substantial long-term follow-up data are needed to identify the potential risk factors for $\mathrm{rLDH}$.

\section{Conclusion}

The current study reported a $7 \%$ rate of $\mathrm{rLDH}$ at the $\mathrm{L} 4 / 5$ level after successful PETD and showed that moderate, but not severe, disc degeneration, small muscle-disc ratio, more fat infiltration of the paravertebral muscles, large sROM, large ISA, and large LL were biomechanical risk factors of recurrent L4-5 disc herniation after PETD. The results also demonstrated that high BMI and heavy physical load intensity, especially work with constant bending posture, increased the possibility of rLDH. We found that the location of disc herniation and foraminoplasty grade had no association with rLDH. In summary, the recurrence of $\mathrm{LDH}$ arises from a combination of factors, and the exact mechanism warrants further investigation.

\section{Ethics and Consent}

All procedures were performed in compliance with the Declaration of Helsinki and were approved by the Ethics Committee of the Affiliated Hospital of Qingdao University. All patients involved gave written informed consent to review their medical records. All personal details were erased before analysis to cover patient data confidentiality.

\section{Acknowledgments}

This work was supported by grants from the National Natural Science Foundation of China (81672200, 81871804 and National Key Research and Development Project (CN) (2019YFC0121400). We are grateful to the Orthopedic Medical Center of the Affiliated Hospital of Qingdao University and the special procedures team.

Meng Kong and Derong $\mathrm{Xu}$ should be considered as co-first authors. 


\section{Disclosure}

The authors declare no relevant financial, personal, political, intellectual or religious conflicts of interests for this work.

\section{References}

1. Eun SS, Lee SH, Sabal LA. Long-term follow-up results of percutaneous endoscopic lumbar discectomy. Pain Physician. 2016;19(8): E1161-e1166.

2. Nie H, Zeng J, Song Y, et al. Percutaneous endoscopic lumbar discectomy for L5-S1 disc herniation via an interlaminar approach versus a transforaminal approach: a prospective randomized controlled study with 2-year follow up. Spine. 2016;41(Suppl 19):B30b37. doi:10.1097/BRS.0000000000001810

3. Cheng J, Wang H, Zheng W, et al. Reoperation after lumbar disc surgery in two hundred and seven patients. Int Orthop. 2013;37 (8):1511-1517. doi:10.1007/s00264-013-1925-2

4. Yin S, Du H, Yang W, Duan C, Feng C, Tao H. Prevalence of recurrent herniation following percutaneous endoscopic lumbar discectomy: a meta-analysis. Pain Physician. 2018;21(4):33 7-350.

5. Choi KC, Lee JH, Kim JS, et al. Unsuccessful percutaneous endoscopic lumbar discectomy: a single-center experience of 10,228 cases. Neurosurgery. 2015;76(4):372-380; discussion 380-371; quiz 381. doi:10.1227/NEU.0000000000000628

6. Li Z, Yang H, Liu M, et al. Clinical characteristics and risk factors of recurrent lumbar disk herniation: a retrospective analysis of three hundred twenty-one cases. Spine. 2018;43(21):1463-1469. doi:10.1097/BRS.0000000000002655

7. Kim JM, Lee SH, Ahn Y, Yoon DH, Lee CD, Lim ST. Recurrence after successful percutaneous endoscopic lumbar discectomy. Minimally Invasive Neurosurgery. 2007;50(2):82-85. doi:10.1055/ s-2007-982504

8. Yao Y, Liu H, Zhang H, et al. Risk factors for recurrent herniation after percutaneous endoscopic lumbar discectomy. World Neurosurg. 2017;100:1-6. doi:10.1016/j.wneu.2016.12.089

9. Hasegawa K, Kitahara K, Hara T, Takano K, Shimoda H, Homma T. Evaluation of lumbar segmental instability in degenerative diseases by using a new intraoperative measurement system. $J$ Neurosurg Spine. 2008;8(3):255-262. doi:10.3171/SPI/2008/8/3/255

10. Kim KT, Lee DH, Cho DC, Sung JK, Kim YB. Preoperative risk factors for recurrent lumbar disk herniation in L5-S1. J Spinal Disord Tech. 2015;28(10):E571-577. doi:10.1097/BSD.000000000 0000041

11. Suk KS, Lee HM, Moon SH, Kim NH. Recurrent lumbar disc herniation: results of operative management. Spine. 2001;26 (6):672-676. doi:10.1097/00007632-200103150-00024

12. Kim HS, You JD, Ju CI. Predictive scoring and risk factors of early recurrence after percutaneous endoscopic lumbar discectomy. Biomed Res Int. 2019;2019:6492675. doi:10.1155/2019/6492675

13. Leijon $\mathrm{O}$, Wiktorin $\mathrm{C}$, Härenstam A, Karlqvist L. Validity of a self-administered questionnaire for assessing physical work loads in a general population. J Occupational Environmental Med. 2002;44 (8):724-735. doi:10.1097/00043764-200208000-00007

14. Pfirrmann CW, Metzdorf A, Zanetti M, Hodler J, Boos N. Magnetic resonance classification of lumbar intervertebral disc degeneration. Spine. 2001;26(17):1873-1878. doi:10.1097/00007632-20010901000011

15. Ranson CA, Burnett AF, Kerslake R, Batt ME, O’Sullivan PB. An investigation into the use of MR imaging to determine the functional cross sectional area of lumbar paraspinal muscles. European Spine J. 2006;15(6):764-773. doi:10.1007/s00586-005-0909-3
16. Battaglia PJ, Maeda Y, Welk A, Hough B, Kettner N. Reliability of the Goutallier classification in quantifying muscle fatty degeneration in the lumbar multifidus using magnetic resonance imaging. J Manipulative Physiol Ther. 2014;37(3):190-197. doi:10.1016/j. jmpt.2013.12.010

17. Kim KT, Park SW, Kim YB. Disc height and segmental motion as risk factors for recurrent lumbar disc herniation. Spine. 2009;34 (24):2674-2678. doi:10.1097/BRS.0b013e3181b4aaac

18. Kim W, Porrino JA, Hood KA, Chadaz TS, Klauser AS, Taljanovic MS. Clinical evaluation, imaging, and management of adolescent idiopathic and adult degenerative scoliosis. Curr Probl Diagn Radiol. 2019;48 (4):402-414. doi:10.1067/j.cpradiol.2018.08.006

19. Wang H, Zhou Y, Li C, Liu J, Xiang L. Risk factors for failure of single-level percutaneous endoscopic lumbar discectomy. J Neurosurg Spine. 2015;23(3):320-325. doi:10.3171/2014.10.SPINE 1442

20. Yu C, Zhan X, Liu C, et al. Risk factors for recurrent L5-S1 disc herniation after percutaneous endoscopic transforaminal discectomy: a retrospective study. Medical Science Monitor. 2020;26:e919888. doi:10.12659/MSM.919888

21. Mimura M, Panjabi MM, Oxland TR, Crisco JJ, Yamamoto I, Vasavada A. Disc degeneration affects the multidirectional flexibility of the lumbar spine. Spine. 1994;19(12):1371-1380. doi:10.1097/ 00007632-199406000-00011

22. Fritz JM, Erhard RE, Hagen BF. Segmental instability of the lumbar spine. Phys Ther. 1998;78(8):889-896. doi:10.1093/ptj/78.8.889

23. Wu J, Zhang C, Lu K, Li C, Zhou Y. Percutaneous endoscopic lumbar reoperation for recurrent sciatica symptoms: a retrospective analysis of outcomes and prognostic factors in 94 patients. World Neurosurg. 2018;109:e761-e769. doi:10.1016/j.wneu.2017.10.077

24. Osti OL, Vernon-Roberts B, Fraser RD. Volvo Award in experimental studies. Anulus tears and intervertebral disc degeneration. An experimental study using an animal model. Spine. 1990;15(8):762-767.

25. Gruber HE, Hanley EN Jr. Observations on morphologic changes in the aging and degenerating human disc: secondary collagen alterations. BMC Musculoskelet Disord. 2002;3:9. doi:10.1186/ 1471-2474-3-9

26. Wiktorin C, Hjelm EW, Winkel J, Köster M. Reproducibility of a questionnaire for assessment of physical load during work and leisure time. Stockholm music i study group. Musculoskeletal intervention center. J Occupational Environmental Medicine. 1996;38 (2):190-201. doi:10.1097/00043764-199602000-00017

27. Seidler A, Bolm-Audorff U, Siol T, et al. Occupational risk factors for symptomatic lumbar disc herniation; a case-control study. Occup Environ Med. 2003;60(11):821-830. doi:10.1136/oem.60.11.821

28. Inglez de Souza M, Ryan R, Ter HG, Packer RMA, Volk HA, De DS. Evaluation of the influence of kyphosis and scoliosis on intervertebral disc extrusion in French bulldogs. BMC Vet Res. 2018;14(1):5. doi:10.1186/s12917-017-1316-9

29. Wei X, Gengwu L, Chao C, et al. Correlations between the sagittal plane parameters of the spine and pelvis and lumbar disc degeneration. J Orthop Surg Res. 2018;13(1):137. doi:10.1186/ s13018-018-0838-6

30. Aikawa T, Shibata M, Asano M, Hara Y, Tagawa M, Orima H. A comparison of thoracolumbar intervertebral disc extrusion in French Bulldogs and Dachshunds and association with congenital vertebral anomalies. Veterinary Surgery. 2014;43(3):301-307. doi:10.1111/j.1532-950X.2014.12102.x

31. Chang HK, Chang HC, Wu JC, et al. Scoliosis may increase the risk of recurrence of lumbar disc herniation after microdiscectomy. J Neurosurg Spine. 2016;24(4):586-591. doi:10.3171/2015.7.SPINE 15133

32. Haher TR, O'Brien M, Dryer JW, Nucci R, Zipnick R, Leone DJ. The role of the lumbar facet joints in spinal stability. Identification of alternative paths of loading. Spine. 1994;19(23):2667-2670 discussion 2671. doi:10.1097/00007632-199412000-00012 
33. el-Bohy AA, Yang KH, King AI. Experimental verification of facet load transmission by direct measurement of facet lamina contact pressure. J Biomech. 1989;22(8-9):931-941. doi:10.1016/00219290(89)90077-8

34. Natarajan RN, Lavender SA, An HA, Andersson GB. Biomechanical response of a lumbar intervertebral disc to manual lifting activities: a poroelastic finite element model study. Spine. 2008;33 (18):1958-1965. doi:10.1097/BRS.0b013e3181822742

35. Serhan HA, Varnavas G, Dooris AP, Patwadhan A, Tzermiadianos M. Biomechanics of the posterior lumbar articulating elements. Neurosurg Focus. 2007;22(1):E1. doi:10.3171/foc.2007.22.1.1

36. Ruetten S, Komp M, Godolias G. An extreme lateral access for the surgery of lumbar disc herniations inside the spinal canal using the full-endoscopic uniportal transforaminal approach-technique and prospective results of 463 patients. Spine. 2005;30(22):2570-2578. doi:10.1097/01.brs.0000186327.21435.cc

37. Tsou PM, Yeung AT. Transforaminal endoscopic decompression for radiculopathy secondary to intracanal noncontained lumbar disc herniations: outcome and technique. Spine J. 2002;2(1):41-48. doi:10.1016/S1529-9430(01)00153-X

38. Zhen L, Shu H, Wen W, Xiao Z. The effect of posterolateral foraminoplasty on the pathoanatomy and biomechanics of lumbar. Chin J Bone Tumor Bone Disease. 2010;9(6).

39. Qiao P, Xu T, Zhang W, Fang Z, Ding W, Tian R. Foraminoplasty affects the clinical outcomes of discectomy during percutaneous transforaminal endoscopy: a two-year follow-up retrospective study on 64 patients. Int $J$ Neurosci. 2020;1-6. doi:10.1080/002074 54.2020 .1732968

40. Zhao R, Yong H, Qun Z. Lumbar biomechanical characteristics after foraminoplasty in different amplitudes: a finite element analysis. J Spinal Surg. 2017;039(010):805-808.

41. Takemitsu Y, Harada Y, Iwahara T, Miyamoto M, Miyatake Y. Lumbar degenerative kyphosis. Clinical, radiological and epidemiological studies. Spine. 1988;13(11):1317-1326. doi:10.1097/00007 632-198811000-00019

42. Lee JC, Cha JG, Kim Y, Kim YI, Shin BJ. Quantitative analysis of back muscle degeneration in the patients with the degenerative lumbar flat back using a digital image analysis: comparison with the normal controls. Spine. 2008;33(3):318-325. doi:10.1097/BRS.0b0 $13 \mathrm{e} 318162458 \mathrm{f}$
43. Yoshihara K, Shirai Y, Nakayama Y, Uesaka S. Histochemical changes in the multifidus muscle in patients with lumbar intervertebral disc herniation. Spine. 2001;26(6):622-626. doi:10.1097/000076 32-200103150-00012

44. Hodges P, Holm AK, Hansson T, Holm S. Rapid atrophy of the lumbar multifidus follows experimental disc or nerve root injury. Spine. 2006;31(25):2926-2933. doi:10.1097/01.brs.0000248453.51 $165.0 \mathrm{~b}$

45. Kalichman L, Hodges P, Li L, Guermazi A, Hunter DJ. Changes in paraspinal muscles and their association with low back pain and spinal degeneration: CT study. European Spine J. 2010;19 (7):1136-1144. doi:10.1007/s00586-009-1257-5

46. Rundell SD, Sherman KJ, Heagerty PJ, Mock CN, Jarvik JG. The clinical course of pain and function in older adults with a new primary care visit for back pain. $J$ Am Geriatr Soc. 2015;63 (3):524-530. doi:10.1111/jgs.13241

47. Cinotti G, Roysam GS, Eisenstein SM, Postacchini F. Ipsilateral recurrent lumbar disc herniation a prospective, controlled study. $J$ Bone Joint Surgery British Volume. 1998;80(5):825-832. doi:10.1302/0301-620X.80B5.0800825

48. Kara B, Tulum Z, Acar U. Functional results and the risk factors of reoperations after lumbar disc surgery. European Spine J. 2005;14 (1):43-48. doi:10.1007/s00586-004-0695-3

49. Huang W, Han Z, Liu J, Yu L, Yu X. Risk factors for recurrent lumbar disc herniation: a systematic review and meta-analysis. Medicine. 2016;95(2):e2378. doi:10.1097/MD.0000000000002378

50. Fiorenza V, Ascanio FJWN. Percutaneous endoscopic transforaminal outside-in outside (TOIO) technique for foraminal and extraforaminal lumbar disc herniations - operative technique. World Neurosurgery. 2019;130.

51. Wu W, Chen Y, Yu L, Li F, Guo W. Coronal and sagittal spinal alignment in lumbar disc herniation with scoliosis and trunk shift. J Orthop Surg Res. 2019;14(1):264. doi:10.1186/s13018-019-1300-0
Risk Management and Healthcare Policy

\section{Publish your work in this journal}

Risk Management and Healthcare Policy is an international, peerreviewed, open access journal focusing on all aspects of public health, policy, and preventative measures to promote good health and improve morbidity and mortality in the population. The journal welcomes submitted papers covering original research, basic science, clinical \& epidemiological studies, reviews and evaluations, guidelines, expert opinion and commentary, case reports and extended reports. The manuscript management system is completely online and includes a very quick and fair peer-review system, which is all easy to use. Visit http://www.dovepress.com/testimonials.php to read real quotes from published authors. 